\title{
Lymphoplasmacytic Lymphoma with Only Lambda Light Chain Monoclonal Paraprotein Expression
}

\author{
Sandhya Cautha, Sorab Gupta, Ahmad Hanif, Valentina Moirangthem, Kevin Jain \\ BronxCare Health System, Bronx, NY, USA
}

Doi: 10.12890/2022_003106 - European Journal of Case Reports in Internal Medicine - @ EFIM 2022

Received: 06/12/2021

Accepted: $12 / 01 / 2022$

Published: $11 / 02 / 2022$

How to cite this article: Cautha S, Gupta S, Hanif A, Moirangthem V, Jain K. Lymphoplasmacytic lymphoma with only lambda light chain monoclonal paraprotein expression. EJCRIM 2022;9: doi:10.12890/2022_003106.

Conflicts of Interests: The authors declare there are no competing interests.

This article is licensed under a Commons Attribution Non-Commercial 4.0 License

\section{ABSTRACT}

Introduction: Lymphoplasmacytic lymphoma (LPL) is a rare low-grade B-cell neoplasm that accounts for approximately $2 \%$ of all haematological malignancies. Most patients have the clinical syndrome of Waldenstrom macroglobulinemia (WM), which is defined as LPL with an associated immunoglobulin M (IgM) serum monoclonal protein. Roughly 5\% of LPL patients secrete non-lgM paraproteins (e.g., IgG, IgA, kappa, lambda) or are non-secretory.

Case description: We report the case of a 41-year-old woman who was diagnosed with non-IgM LPL with lambda light chain monoclonal paraprotein production and normal serum immunoglobulin levels. The MYD88 L265P mutation was detected on fluorescence in-situ hybridization (FISH) analysis of the bone marrow. The patient underwent treatment with a combination of ibrutinib and rituximab. There was an initial response but she died 8 months after diagnosis.

Discussion: Non-IgM LPL poses diagnostic and therapeutic challenges to clinicians as it is an exceptionally rare malignancy with a heterogeneous clinicopathological presentation and scarce literature. Among non-IgM LPL cases, those with lambda light chain production are even more rare. To the best of our knowledge, none have been reported to date. The addition of MYD88 L265P testing to the diagnostic armamentarium of non-IgM LPL cases is advisable for potential therapeutic reasons.

\section{LEARNING POINTS}

- Our case report and literature review provide insight into non-IgM lymphoplasmacytic lymphoma (LPL), an extremely rare malignancy.

- Our case report highlights the importance of the need for new treatments for non-IgM LPL.

\section{KEYWORDS}

Lymphoplasmacytic lymphoma, non-IgM, Waldenstrom macroglobulinemia, lambda light chains, MYD88 L265P, ibrutinib, rituximab

\section{CASE DESCRIPTION}

A 41-year-old woman was referred to our haematology clinic with complaints of progressively worsening painful bruising, tingling sensations in the extremities, fatigue, night sweats, loss of weight, and loss of appetite for 8 months. Physical examination revealed tachycardia and multiple bruises over the extremities. The laboratory findings were as follows: white blood cells (WBC) 18,000/ $\mu$ l (53\% lymphocytes,

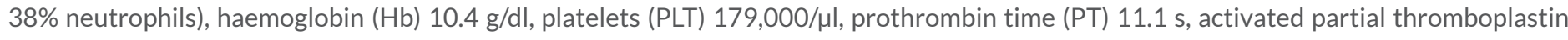
time (APTT) 32.4 s, 32 -microglobulin 2.8 mg/l, lactate dehydrogenase (LDH) $233 \mathrm{U} / \mathrm{l}$, lgM 138 mg/dl, lgG 806 mg/dl, lgA 39 mg/dl, free lambda serum $1425 \mathrm{mg} / \mathrm{l}$, free kappa serum $6.6 \mathrm{mg} / \mathrm{l}$, and free kappa/lambda ratio <0.01. Normal results were obtained for fibrinogen, factor VIII, X and XIII assays, von Willebrand factor (VWF) antigen, VWF multimer assay, VWF-ristocetin cofactor assay, C3 and C4 levels, 
and creatinine clearance. Infective and auto-immune work-up was negative. Serum protein electrophoresis showed a selective protein loss pattern, and the serum immunofixation test showed a faint band in the lambda region. Urine electrophoresis showed monoclonal protein with a protein-creatinine ratio of $2757 \mathrm{mg} / \mathrm{g}$, and the urine immunofixation test showed a free monoclonal lambda band. Prominent axillary lymphadenopathy was noted on computed tomography (CT). Peripheral blood flow cytometry showed atypical B-cells which were CD19+, CD20+, FMC7+, CD5-, CD10-, CD23-, CD103-. The bone marrow biopsy exhibited malignant non-Hodgkin lymphoma, low grade, B-cell type with 100\% tumour infiltration of the marrow core. Immunohistochemical studies demonstrated CD19+, CD20+, FMC7+, CD5-, CD10-, CD23-, CD11c-, CD103- B-cells that comprised $74 \%$ of total cells and a small population ( $<1 \%$ of total cells) of plasma cells brightly positive for CD38 with cytoplasmic lambda light chain restriction, confirming a diagnosis of LPL with lambda light chain monoclonal protein. Similar results were obtained from the lymph node biopsy. Abdominal fat pad biopsy for amyloid was negative by Congo Red histochemistry. The MYD88 L265P mutation was detected on fluorescence in-situ hybridization (FISH) analysis of the marrow sample. Immunoglobulin gene re-arrangement, IgH/MAF t(14;16), IgH/MAF t(14;20), TP53 deletion, and chromosome 13q deletion were negative. NCCN guidelines for the lymphoplasmacytic disorder were reviewed for treatment decisions. Based on a phase 3 trial of the iNNOVATE Study Group, treatment with ibrutinib and rituximab was recommended in accordance with category 1 evidence. The patient was treated with rituximab $375 \mathrm{mg} / \mathrm{m}^{2}$ weekly for 4 weeks and ibrutinib $480 \mathrm{mg}$. However, ibrutinib had to be stopped for 20 days as she developed upper gastro-intestinal bleeding (a known side-effect). It was resumed at a dose of $140 \mathrm{mg}$ and later increased to $280 \mathrm{mg}$ with good tolerance. After 6 weeks of treatment, the lambda light chain levels dropped to $50 \%$ (720 mg/l), symptomatic improvement was noted, and axillary lymphadenopathy was resolved. The patient achieved a partial response (PR) according to the 6th International Workshop on Waldenstrom macroglobulinemia response criteria. However, 8 months after diagnosis she was admitted with fever and bloody diarrhoea and was found to have reduced ejection fraction heart failure with pleural and pericardial effusions. Ibrutinib was withheld as it can cause bleeding and heart failure. The patient developed Escherichia coli sepsis with severe acute kidney injury requiring haemodialysis and subsequently died.

\section{DISCUSSION}

Lymphoplasmacytic lymphoma (LPL, previously called lymphoplasmacytoid lymphoma) is an uncommon low-grade B-cell neoplasm exhibiting a spectrum of B-cell differentiation ranging from small lymphocytes to plasma cells ${ }^{[1]}$. LPL accounts for approximately $2 \%$ of all haematological malignancies and 1000-1500 new cases are diagnosed every year in the USA ${ }^{[2-4]}$. Most patients have the clinical syndrome of Waldenstrom macroglobulinemia (WM), which is defined as LPL with an associated immunoglobulin M (IgM) serum monoclonal protein ${ }^{[1]}$. Roughly $5 \%$ of LPL patients secrete non-IgM paraproteins (e.g., IgG, IgA, kappa, lambda) or are non-secretory. The clinical presentation in LPL is varied and can include symptoms related to tumour infiltration or monoclonal protein production. The diagnosis of LPL is typically made by exclusion of other small B-cell lymphoid neoplasms with plasmacytic differentiation. On immunophenotyping, the lymphocytes were CD5+/-, CD10-, CD19+, CD20+, CD22+, CD23-, CD25+, FMC7+, CD103- and plasma cells were CD38+, CD138+ and CD45-. However, accurate diagnosis of LPL can be difficult because variations from the typical morphology and immunophenotype can occur [5]. Recently, whole-genome sequencing studies have identified mutations in the MYD88 gene (MYD88 L265P) in 90\% of LPL cases, and testing for this abnormality offers an appealing diagnostic testing aid in difficult-to-classify cases ${ }^{[6]}$.

Further evaluation of these cases includes serum protein electrophoresis to look for the presence of a monoclonal protein spike. Cases of LPL not secreting an IgM monoclonal protein are uncommon and have been reported infrequently ${ }^{[7-11]}$. These cases more commonly present with B symptoms and extramedullary disease, and have higher $\beta 2$-microglobulin and LDH levels when compared with IgM LPL patients ${ }^{[7-11]}$. While some patients are asymptomatic, symptoms related to anaemia, neuropathy, elevated creatinine, bleeding diathesis and recurrent infections can be present ${ }^{[10,11]}$. Higher mortality in the first year after diagnosis and worse overall survival, with no difference in progression-free survival, was observed in these patients when compared with IgM LPL patients ${ }^{[7,10]}$. The presence of a MYD88 mutation in these cases, although at a lower rate, suggests that these may in fact be biologically related to IgM LPL/WM ${ }^{[8]}$. Based on this, we postulate the treatment for these tumours is, therefore, similar to that for WM. Treatment should be started only in those who are symptomatic. Importantly, high paraprotein levels per se should not be regarded as a criterion for therapy initiation. The treatment options for LPL are heterogeneous and there are no prospective studies on which to base a standard treatment regimen. Available evidence supports rituximab alone or rituximab-based regimens as effective first-line or salvage therapy ${ }^{[7]}$. Among these, bendamustine-rituximab and bortezomib-dexamethasone-rituximab combinations were associated with higher response rates and longer survival when compared with cyclophosphamide-dexamethasone-rituximab ${ }^{[12,13]}$. Ito et al. reported successful treatment of two MYD88 L265P-mutation-negative nonIgM LPL cases with bortezomib-containing regimens ${ }^{[14]}$. In patients with the MYD88 L265P mutation, ibrutinib is an effective treatment option as this mutation triggers survival signalling through BTK and HCK, and the cell lines expressing it undergo apoptosis in response to ibrutinib, which targets both of these kinases ${ }^{[15]}$. The phase 3 iNNOVATE trial compared patients with LPL treated with ibrutinib plus rituximab or rituximab plus placebo. At 30 months of follow-up, ibrutinib plus rituximab treatment showed an overall response rate of $95 \%$ 
versus $48 \%$, and a progression-free survival rate of $78 \%$ versus $41 \%$ with rituximab plus placebo treatment ${ }^{[16,17]}$.

Ours is a very rare case presentation of a non-IgM LPL with only lambda light chain monoclonal paraprotein production and normal serum immunoglobulin levels. In congruence with the existing data, our patient had B symptoms, high $\beta 2$-microglobulin and LDH levels, and biopsy-proven extramedullary disease. The diagnosis was made by a bone-marrow biopsy showing tumour infiltration by B-cells positive for CD 19, CD20, FMC7, and a small population of CD38+ plasma cells with cytoplasmic lambda light chain restriction. Serum and urine immunofixation showed a monoclonal lambda band, and FISH analyses detected the MYD88 L265P mutation. This patient was treated with four weekly cycles of rituximab $\left(375 \mathrm{mg} / \mathrm{m}^{2}\right)$ and ibrutinib (dose adjusted to side effects). She reported symptomatic improvement with treatment initiation and by day 42 , serum lambda light chain levels had dropped by $50 \%$ ( $720 \mathrm{mg} / \mathrm{l}$ ) with radiological resolution of axillary lymphadenopathy. However, she died 8 months after diagnosis and the outcome can be attributed to disease progression and/or ibrutinib. To summarize, patients with this disease have a poor prognosis.

\section{CONCLUSION}

Non-IgM LPL poses diagnostic and therapeutic challenges to clinicians as it is an extremely rare malignancy with a heterogeneous clinicopathological presentation and scarce literature. Among the non-lgM LPL cases, cases with only lambda light chain production are even more rare, and to the best of our knowledge, none have been reported to date. Our case report will be a valuable addition to the literature of this extremely rare entity and help guide the management of such cases in the future. The addition of MYD88 L265P testing to the diagnostic armamentarium of non-IgM LPL cases is advisable for potential therapeutic reasons.

\section{REFERENCES}

1. Swerdlow S, Harris NL, Jaffe ES, Pileri SA, Stein H, Thiele J, eds. WHO classification of tumours of haematopoietic and lymphoid tissues. 4 th ed. Lyon, France; IARC; 2008.

2. Swerdlow SH, Campo E, Pileri SA, Harris NL, Stein H, Siebert R, et al. The 2016 revision of the World Health Organization classification of lymphoid neoplasms. Blood 2016 May 19;127(20):2375-2390.

3. Castillo JJ, Ghobrial IM, Treon SP. Biology, prognosis, and therapy of Waldenström macroglobulinemia. Cancer Treat Res 2015;165:177-195.

4. Yun S, Johnson AC, Okolo ON, Arnold SJ, McBride A, Zhang L, et al. Waldenström macroglobulinemia: review of pathogenesis and management. Clin Lymphoma Myeloma Leuk 2017 May 1;17(5):252-262.

5. Naderi N, Yang DT. Lymphoplasmacytic lymphoma and Waldenström macroglobulinemia. Arch Pathol Lab Med 2013 Apr;137(4):580-585.

6. Treon SP, Xu L, Yang G, Zhou Y, Liu X, Cao Y, et al. MYD88 L265P somatic mutation in Waldenström's macroglobulinemia. N Engl J Med 2012 Aug 30;367(9):826-833.

7. Cao X, Medeiros LJ, Xia Y, Wang X, Thomas SK, Loghavi S, et al. Clinicopathologic features and outcomes of lymphoplasmacytic lymphoma patients with monoclonal IgG or IgA paraprotein expression. Leuk Lymphoma 2016 May 3;57(5):1104-1113.

8. King RL, Gonsalves WI, Ansell SM, Greipp PT, Frederick LA, Viswanatha DS, et al. Lymphoplasmacytic lymphoma with a non-lgM paraprotein shows clinical and pathologic heterogeneity and may harbor MYD88 L265P mutations. Am J Clin Pathol 2016 Jun 1;145(6):843-851.

9. Varettoni M, Boveri E, Zibellini S, Tedeschi A, Candido C, Ferretti VV, et al. Clinical and molecular characteristics of lymphoplasmacytic lymphoma not associated with an IgM monoclonal protein: a multicentric study of the Rete Ematologica Lombarda (REL) network. Am J Hematol 2019;94(11):1193-1199.

10. Kang J, Hong JY, Suh C. Clinical features and survival outcomes of patients with lymphoplasmacytic lymphoma, including non-IgM type, in Korea: a single-center experience. Blood Res 2018 Sep 31;53(3):189-197.

11. Itchaki G, Dubeau T, Keezer A, Meid K, Xu L, Yang G, et al. Non-IgM secreting lymphoplasmacytic lymphoma-experience of a reference center for Waldenstrom macroglobulinemia. Blood 2018 Nov 29;132:2886.

12. Castillo JJ, Gustine JN, Meid K, Dubeau TE, Severns P, Xu L, et al. Response and survival for primary therapy combination regimens and maintenance rituximab in Waldenström macroglobulinaemia. Br J Haematol 2018 Apr;181(1):77-85.

13. Paludo J, Abeykoon JP, Shreders A, Ansell SM, Kumar S, Ailawadhi S, et al. Bendamustine and rituximab (BR) versus dexamethasone, rituximab, and cyclophosphamide (DRC) in patients with Waldenström macroglobulinemia. Ann Hematol 2018 Aug;97(8):1417-1425.

14. Ito K, Nishiyama R, Hirano K, Yamada K, Sekiguchi N. Successful treatment of non-IgM lymphoplasmacytic lymphoma by bortezomib-containing regimen: case reports and review of literature. Blood Res 2019 Sep 1;54(3):236-240.

15. Castillo JJ, Ghobrial IM, Treon SP. Response to ibrutinib in a patient with IgG lymphoplasmacytic lymphoma carrying the MYD88 L265P gene mutation. Leuk Lymphoma 2016 Nov 1;57(11):2699-2701.

16. Dimopoulos MA, Tedeschi A, Trotman J, García-Sanz R, Macdonald D, Leblond V, et al. Phase 3 trial of ibrutinib plus rituximab in Waldenström's macroglobulinemia. N Engl J Med 2018 Jun 21:378(25):2399-2410.

17. Buske C, Tedeschi A, Trotman J, García-Sanz R, MacDonald D, Leblond V, et al. Ibrutinib treatment in Waldenström's macroglobulinemia: follow-up efficacy and safety from the iNNOVATETM study. Blood 2018 Nov 29;132:149. 